\title{
The challenge of explaining new physics concepts and phenomena
}

\author{
Eirik Gramstad ${ }^{* \dagger}$ \\ University of Oslo \\ P.O.Box 1048. 0316 Oslo, Norway \\ E-mail: egramsta@cern.ch
}

\begin{abstract}
With the advent of higher energies and higher collision rates, the LHC continues the exciting voyage towards new physics, allowing physicists all over the world to explore a previously unknown territory full of promise. So far the International Particle Physics Outreach Group (IPPOG) international masterclass developers, with the help of physicists and in close contact with teachers, have been successful in designing educational material and in engaging high school students to work, with real LHC data, on current hot topics, such as the discovery of the Higgs boson. One of the current challenges is to convey advanced physics concepts and to introduce new ideas beyond today's theoretical framework describing the content of the Universe and its evolution. How can we influence the teaching at schools in order to provide a better basis for attending masterclasslike events, and in general for understanding experimental results and new theoretical ideas? An IPPOG initiative deals with effective ways of explaining new physics. Moreover, physicists, in close contact with high school teachers and university departments of education, are investigating a more professional and research-based view on methods and ideas for introducing and explaining new physics concepts. A program plan, together with relevant material, must be created and incorporated to suit the high school curriculum and even replace the ordinary text book on the subject. This is crucial in order to enable us to explain new physics concepts and related enigmas such as dark matter, the role of gravity at the quantum scale, the possible unification of all fundamental forces and the physics of the early Universe.
\end{abstract}

The European Physical Society Conference on High Energy Physics

5-12 July, 2017

Venice

\footnotetext{
*Speaker.

${ }^{\dagger}$ with Farid Ould-Saada and Magnar K. Bugge - on behalf of the International Particle Physics Outreach Group
} 


\section{Introduction}

The International Particle Physics Outreach Group (IPPOG) has developed an educational activity, the International Masterclasses (IMC) [1], that brings the excitement of cutting-edge particle physics research into the classroom. Each year, since 2005, thousands of high school students in many countries all over the world come to nearby universities or research centers for one day in order to unravel the mysteries of particle physics and be scientists for a day. The LHC data have been successfully deployed since 2010 in IMC where students perform various measurements based on proton-proton, lead-lead and proton-lead collisions. The Higgs discovery, the investigation of the quark-gluon plasma phenomenon and other measurements and scientific methods could be shared with the students. [2, 3, 4, 5, 6, 7, 8, 9, 10, 11, 12]

However, the recent release of real LHC collision data through the CERN Open Data Portal [13] has triggered an extension of the educational material to be used at the IMC and at universities to prepare for whatever will be discovered in the coming years and to give a more researchbased approach in introducing particle physics through hands-on activities [14].

\section{The Z-path masterclass event}

The Z-path $[5,15,16]$ is the most popular educational tool which is part of the IMC. In this measurement students identify particles (electrons, muons and photons) and calculate the invariant mass of different pairs of these particles by analyzing real proton-proton collision events recorded by the ATLAS detector $[17,18]$ at the LHC. A typical IMC day starts with a set of lectures covering all what the students need to know to perform the practical session later in the day. The idea is that the Masterclasses should be a self-contained program requiring only some basic physics knowledge. ${ }^{1}$ This obviously makes the Masterclass programs quite ambitious, but give at the same time the students an unique opportunity to participate in hands-on activities at the forefront of modern high energy physics. However, the current Z-path measurement as well as the data released thorough the CERN Open Data Portal have still a huge potential in terms of giving students access to very interesting aspects of particle physics. The question is if the students have the background and means needed to understand and utilize the full potential these tools give.

\section{Important concepts to master the Z-path (and other LHC-physics activities)}

When the students come to the Masterclasses we start from the fundamental pieces of the Universe and define the four fundamental forces and the intrinsic properties of particles. First the familiar concept of charge is introduced. Charge can be measured by looking at how the particle trajectories bend when moving inside a magnetic field. We then introduce the concept of mass, which from the measurements of the energy and momentum of the decay particles easily can be calculated for any unstable particle produced in the collisions. This is what the students use later when performing the practical measurement. Finally we introduce spin, which for almost everybody is a totally new concept that the students have a priori no experience with (at least in

\footnotetext{
${ }^{1}$ a typical IMC participant is a high school student with some specialization in physics in his/her final years before entering University or College.
} 
a particle context). This is probably due to the fact that the spin of a particle is a pure quantum mechanical property with no classical equivalent. However, it is not so different from electric charge, since the spin of a particle leads to important spin-offs and macroscopic effects measurable by using magnetic fields. ${ }^{2}$ We do not go into all the details about spin, but try to convince the students that this is a very important property which we can, and do, measure (just like electric charge and mass). Equipped with these important concepts we introduce the Standard Model of particle physics by grouping the particles based on their intrinsic properties and symmetry patterns, in much the same way as the well known periodic table of elements. An example on how this is done in the Z-path lectures is shown in Figure 1. By coupling the already introduced electric charge

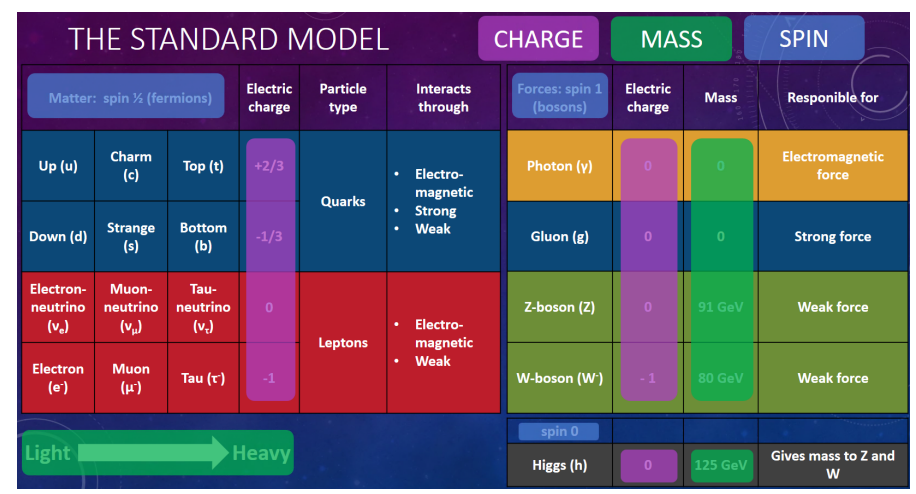

Figure 1: How the Standard Model of particle physics is introduced to the students in the Z-path Masterclasses. Fundamental particles and forces are classified according to intrinsic properties of particles, following symmetry patterns leading to various conservation laws.

to electromagnetism one could extend the same concept to the weak and strong forces by presenting two other charges, i.e. weak and color charge. Currently this is not part of the topics covered in IMC's Z-path program. Nevertheless, this would indeed present a more complete and fundamental understanding of the Standard Model in terms of symmetries and conservation laws.

\section{Expanding the program}

In the Z-path the students identify electrons, muons and photons and look for events where there are either two leptons, two photons or four leptons. This is motivated by the fact that many well known, but also hypothetical particles, decay to these final states. For each collision event the students go through, they calculate the invariant mass of the set of particles they have identified. To prepare the students for new discoveries there are some simulated events of hypothetical particles mixed into the real LHC data. This is done without notifying the students in advance. The simulated particles are: a new weak force particle, $\mathrm{Z}$, with a mass of $1 \mathrm{TeV}$ and an excitation of the Graviton, the hypothetical mediator of the gravitational force, with mass $1.5 \mathrm{TeV}$. Combining the results across all the participating institutes ${ }^{3}$ allows high statistics distributions of the different channels (di-lepton, four-lepton and di-photon) at low (0-200 GeV) and high mass (800-1800 GeV)

\footnotetext{
${ }^{2}$ the Stern-Gerlach experiment

${ }^{3}$ Each institute receive a complementary data set not overlapping with any of the other participating institutes' data sets.
} 
to be plotted, as depicted in Figure 2. These invariant mass distributions, which carry the signature

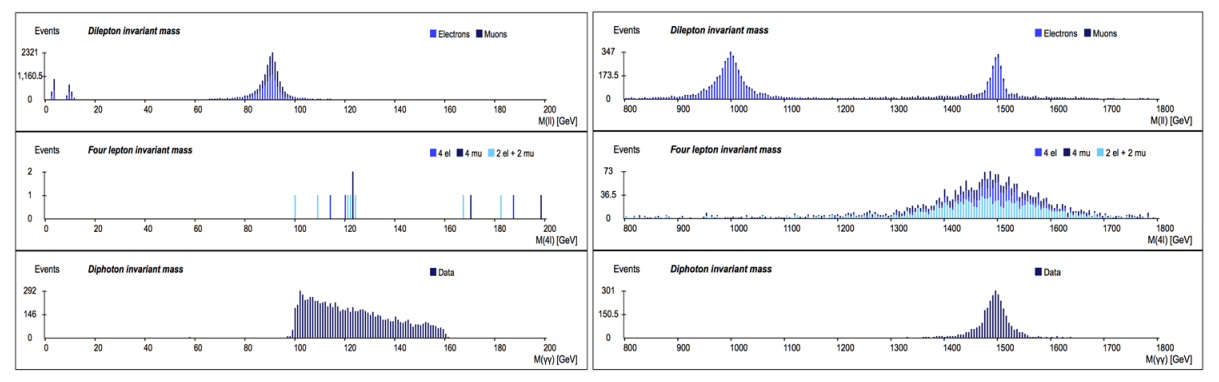

Figure 2: The invariant mass distributions obtained when analyzing all the data available for the Z-path exercise. From top to bottom are the di-lepton, four-lepton and di-photon distributions. To the left are the low mass resonances $(0-200 \mathrm{GeV})$ and to the right are the high mass resonances $(800-1800 \mathrm{GeV})$. Plots made with OPloT [19].

of known and not yet discovered particles, initiate several discussions, both locally at the institutes and in the final video conference with CERN and the other participating institutes. For the Z-path Masterclasses the most important topics are: the concept of statistics and statistical significance, i.e. the fact that combining each individual student's results with all the others helps clearer structures to appear, allowing to draw conclusions and measure properties such as the mass of known particles. Secondly, the unexpected peaks in the invariant mass distributions is a proof of concept of how we actually search for new particles/resonances in distributions of the invariant mass. This was exactly how the Higgs boson was discovered in 2012 [20]. Through these measurements the IMC give students a real taste on high energy particle physics research and how they can master exactly the same methods as are used to discover and search for new particles. This has made the IMC an unique and extremely successful program in introducing particle physics to high school students and in attracting them to study physics.

Nevertheless, taking a closer look at the high mass region of the distributions reveals several other interesting features, e.g. the $1 \mathrm{TeV}$ resonance only appearing in the di-lepton plot while the 1.5 TeV resonance appears in the di-photon and four-lepton plots in addition. This has of course to do with the spin of the hypothetical particle and the fact that the $Z^{\prime}$ has spin 1 and a Graviton has spin 2. In this sense, the information carried by Figure 2 is valuable and helpful for students to indirectly sense the behavior of particles with different spin properties.

To push the discussions further along this path within the scope of the IMC would involve more time than the day allocated and several new concepts to be introduced more thoroughly to the students. If this is done in an understandable and simplified way it would give the opportunity to understand and discuss other interesting aspects of particle physics. A way to measure and gain information about spin would be to for instance study further the decay angular distribution of the hypothetical particles. A better understanding of the concept of spin could open up for a better understanding of many important topics such as the spin-symmetry (matter-force/fermion-boson) which sets the basis for supersymmetry, the properties of the particles and their role in the creation and evolution of the early universe, how to incorporate gravity into the particle physics framework and to better understand important macroscopic phenomena such as the periodic table of elements and lasers. 
Spin is only one example on how we, by giving a better understanding of the fundamental concepts in particle physics, can improve and extend events such as Masterclasses rendering them even more interesting, comprehensive and attractive. There are many more concepts to bring in if we want to go beyond buzz-words in the way we teach young people about particle physics. To achieve this some simplification, reorganization and extension of the existing educational material based on the existing measurements already performed in any of the IMC measurements could be beneficial. IPPOG is putting efforts to sustain these programmes and extend them in various directions.

\section{How to make the event more learning-effective and attractive?}

The IMC programme has been extremely successful and popular, with the number of students, institutes and countries taking part in the many events steadily increasing. However, there is room for improvement and extensions. For most of the students everything which is presented at the IMC is new. A full bottom to top introduction is impossible to give in a 2 hours lecture. Our experience from the past years tells us that the optimal would be if the students had already worked on some of the topics prior to the IMC participation. There exists already a web-page [5] which can be used before and/or after the Masterclass. However, this page is not self-contained and not very widely used and the material is sometimes hard to grasp, even for the teachers. That is why we want to extend and improve the educational material such that students at high schools can get a better knowledge of particle physics and thereby open up for more interesting discussion, not just within the Masterclasses, but also in other contexts where real LHC data released through CERN Open Data Portal is used. By making use of the real data and in close connection with high school teachers, experts in education and didactic we hope to influence how the field of particle physics is taught, not just in high schools, but also in introductory and advanced courses in particle physics at the universities. ${ }^{4}$

\section{Conclusions, plans and outlook}

Through the experience with the Z-path, the IMC and the recent use of the LHC data released through the CERN Open Data Portal we have been inspired to try and extend the educational material, in order to be able to follow the LHC heartbeats, share whatever LHC and future experiments will discover and better support both theoretical and experimental lectures at universities and high schools. The real data being released enables new ways to introduce difficult concepts in particle physics. Collaboration with high school teachers, university departments of education and experts in didactic have already been established in order to investigate new ways to present the important concepts. In parallel, fruitful discussions are ongoing within IPPOG on the same topics. We have started to further develop tools and material to be used in IMC and beyond. Goal is to include the use of real LHC data in particle physics courses and the universities as well as develop and extend the Z-path to be an self-contained resource to be used at high schools, not only as part of the IMC.

\footnotetext{
${ }^{4}$ The Experimental Particle Physics group at the University of Oslo, active member of IPPOG, received in 2017 a grant of $150 \mathrm{k} €$ over three years from Thon Stiftelsen to develop and extend the Z-path further: https: //www . mn. uio.no/fysikk/english/research/projects/zpath/index.html
} 


\section{References}

[1] “LHC@InternationalMasterclasses."

http: //atlas.physicsmasterclasses.org/en/index.htm.

[2] International Masterclasses in the LHC era, CERN Courier (May, 2014) .

[3] Reaching out with particle physics, CERN Courier (2015) .

[4] Reaching out in the Era of Big Science, CERN Courier (2017) .

[5] “Atlas Z-path web page." http: / / atlas.physicsmasterclasses.org/en/zpath.htm.

[6] "Atlas W-path web page."

http://atlas.physicsmasterclasses.org/en/wpath.htm.

[7] "CMS WZH-path web page."

http://cms . physicsmasterclasses.org/pages/cmswz.html.

[8] “J/ $\psi$-path web page."

http://cms . physicsmasterclasses.org/pages/cms jpsi.html.

[9] “ALICE measurement web page." http://www-alice.gsi.de/masterclass/.

[10] “ALICE ROOT-based web page."

http://aliceinfo.cern.ch/public/MasterCL/MasterClassWebpage.html.

[11] "LHCb measurement web page." http: / / lhcb-public.web.cern. ch/lhcb-public/ en/LHCb-outreach/masterclasses/en/.

[12] "TOTEM Masterclass web page."

http://totem-experiment.web.cern.ch/totem-experiment/education/.

[13] “CERN OpenData Portal.” http://opendata.cern.ch/.

[14] Ould-Saada, Farid, Bringing LHC data into the classroom, in proceedings of ICHEP2012, PoS ICHEP2014 (2015) (2015) .

[15] M. Pedersen, F. Ould-Saada and M. K. Bugge, Sharing ATLAS data and research with young students, Tech. Rep. ATL-OREACH-PROC-2015-001, CERN, Geneva, Feb, 2015.

[16] Bugge, Magnar K., Gramstad, Eirik, Morisbak, Vanja, Ould-Saada, Farid, Pedersen, Maiken and Raddum, Silje H., ATLAS masterclasses - $W$ and $Z$ path physics and presentation of the $Z$ path measurement, EPJ Web of Conferences 71 (2014) 00024.

[17] ATLAS collaboration, M. Capeans, G. Darbo, K. Einsweiller, M. Elsing, T. Flick, M. Garcia-Sciveres et al., ATLAS Insertable B-Layer Technical Design Report, Tech. Rep. CERN-LHCC-2010-013. ATLAS-TDR-19, Sep, 2010.

[18] ATLAS collaboration, The ATLAS Experiment at the CERN Large Hadron Collider, Journal of Instrumentation 3 (2008) S08003.

[19] V. Morisbak et al., "OPloT, Oslo Plotting Tools." http: / / cernmasterclass.uio. no/.

[20] ATLAS collaboration, Observation of a new particle in the search for the Standard Model Higgs boson with the ATLAS detector at the LHC, Physics Letters B 716 (2012) 1 - 29. 